\title{
Design of a Calorimetric Test Facility to Replicate Real Boundary Conditions in the Gulf Countries
}

\author{
Roberto Garay-Martinez ${ }^{1, *}$, Bejay Jayan ${ }^{2}$, and Beñat Arregi ${ }^{1}$ \\ ${ }^{1}$ TECNALIA, Basque Research and Technology Alliance (BRTA), 48160 Derio, Spain \\ ${ }^{2}$ Smart Insulation Finishing Systems, Aspect tower D 805, Business Bay, PO Box 125392, Dubai, United Arab Emirates
}

\begin{abstract}
The design and modelling of a calorimetric test infrastructure for building envelopes is performed for the side-by-side assessment of different building envelope systems. The infrastructure is designed for representing transient weather conditions in Middle east. It consists of 3 "cold" experimental chambers and a larger "hot" experimental chamber. All three cold chambers have one equally sized envelope element exposed to the larger chamber. The test facility is designed to allow testing on walls and roofs, where different envelope insulation systems will be installed over a common substrate. Heating and cooling loads of all experimental chambers are calculated, and systematic load differences assessed. Heat flow across test samples and other surfaces in the test are calculated. Insulation levels of envelope surfaces in experimental chambers are specified to provide a good match between heat transfer across test samples and heat input to experimental chambers.
\end{abstract}

\section{Introduction}

According to the Sustainable Building and Climate Initiative of the UN [1], buildings are responsible for $40 \%$ of the global primary energy consumption. A large amount of this is required for space conditioning (heating and cooling).

Roof, façades and windows are the physical interface between indoor and outdoor conditions. Heat transfer across these components is responsible for over $60 \%$ of heat transfer in non-insulated buildings. Furthermore, their performance substantially defines the heat dynamics of buildings. It is considered that there is a great heat flux reduction potential by incorporating additional insulation to building envelopes.

The installation of External Thermal Insulation Composite Systems (ETICS) in existing buildings is increasingly common in modern societies. This is also enforced by public policy [2, 3, 4] and building codes [5, 6] in many countries. In these codes, requirements are imposed to thermal performance metrics of envelopes, commonly assessed against international standards [7, 8, 9] to facilitate the introduction of building envelope insulation systems.

Energy efficiency codes or green building regulations for public/private buildings started to be implemented across many states of UAE from 2010. Among the seven states, Abu Dhabi [10], Dubai [6], and Sharjah were some of the first to implement these regulations and consequently make them mandatory. The regulations cover many areas such as ecology and planning, building envelopes, conservation and efficient energy systems, resource effectiveness and waste management. Steps were particularly taken to improve the building envelope, with the implementation of stringent thermal performance requirements ( $U$-values) for walls, roofs and windows.

Considering the relatively shorter experience, there is a need for an integrated approach towards the construction sector for the incorporation of building envelope insulation systems. This approach should consider the design and validation of thermal insulation systems specifically designed for integration with local architectural systems, and operation under extreme conditions - at high temperature, large daily thermal amplitude and solar irradiation levels. Also, sectorial awareness and engagement activities and installer training activities are needed. In broad terms, there is a need for the construction sector in UAE and Middle East to make their own path, as it has been done in cold climates such as EU and North America since the oil crisis. This should always be made considering the local specificities in terms of climate, architecture, etc.

With regards to thermal performance, there is a need for the proper design and testing of adapted solutions. Within the Gulf countries, relevance is given to fire performance [11], which implies substantial modification to material selection and system design. Performance assessment needs to be guaranteed by means of heat transfer assessment processes typical to construction materials [12], but also at a real scale under transient boundary conditions typical of the Gulf countries.

Experimentation in building physics is commonly divided in on-site and laboratory assessment methods, where methods have been highly normalized $[8,12]$. As

* Corresponding author: roberto.garay@tecnalia.com 
an intermediate step, several research facilities have been developed for envelope testing under transient boundary conditions. [13] presents a review on many available test facilities.

In all the aforementioned procedures an underlying assumption is made in relation to the heat transfer directionality. Indoor-to-outdoor heat transfer is assessed, due to the greater research and standardization tradition in cold climates. Equally, a predominance of steady-state heat transfer is expected with relatively reduced transient ambient conditions.

These assumptions are under discussion in some Mediterranean countries, where the relevance of climate and building dynamics increases, and alternated heating and cooling loads can be present on the same day.

Considering the extreme hot climate in the Gulf countries, a specific redefinition of assessment methods is required. Steady-state thermal performance is again relevant, but the directionality of heat transfer is opposite to the one established in the available standards and test methods. Also, climate conditions are highly transient due to the great impact of solar radiation both in ambient temperature and direct incidence in building envelopes.

Dynamic assessment methods were developed in PASSYS/PASLINK test environments [14]. Some of these systems are located under Mediterranean climatic conditions (Almeria), and have already performed studies under hot and highly dynamic weather.

Many recent references provide insights to the benefits of transient assessment methods [15, 16, 17] and experimental campaign lengths [18]. Depending on the case, inductive knowledge determines that robust performance data can be provided with campaign lengths in the range of 5 to 10 days.

Considering the aforementioned issues, there is a need to develop specific performance assessment capacities in the Gulf countries, focusing at its particular specificities. Relevant recent references [19, 20, 21] have proven that successful infrastructures require not only the test apparatus/building/device itself, but also a careful selection of assessment methods and highly specialized training of staff.

In this paper, a novel test facility for full scale testing of building envelope systems under realistic boundary conditions is presented. This facility is owned and operated by Smart Insulation Finishing Systems (SIFS) [22]. SIFS is a manufacturer of innovative insulation products for the building envelope in the Middle East, offering sustainable and practical solutions to help reduce energy losses in buildings. As part of its key business processes SIFS has developed consultancy and on-site testing capacity, which will be now complemented with a singular permanent facility. This facility is envisioned as the cornerstone of its in-house product development \& testing capacities, and will be used for demonstration and capacity building.

The facility comprises 3 calorimetric chambers and a large hall. All chambers have two surfaces (one wall and one roof) exposed to the large hall. The large hall will be conditioned to characterize external weather, while the chambers will be kept at indoor comfort conditions.

\section{Purpose of the test facility}

The purpose of the test facility is the comparative assessment of building envelope insulation systems. The assessment is performed by means of two sets of performance metrics:

- Performance assessment based on standardized metrics. $U$-value measurements will be performed based on ISO [8] and ASHRAE [9] standards.

- Performance assessment based on non-standardized metrics. Cooling load assessment of experimental chambers based on heat meters will be performed. Also, transient heat transfer assessment will be performed inspired by [23].

The test facility focuses on comparative parallel testing of various envelope systems, which can be measured simultaneously in adjacent chambers under relatively short measurement campaigns. Experimental sequence length is estimated to be in the range of 7 to 10 days.

Test specimens are exposed to transient boundary conditions. This is specifically relevant with regards to the outdoor boundary conditions, where the temperature in the large hall will be set under harmonic conditions, equivalent to outdoor climate in UAE or any other Middle East country.

The facility is targeted at the $R \& D$ and demonstration activity at SIFS and located within its premises in UAE.

\section{Climate conditions}

Following the widely used Köppen-Geiger climate classification, based on average monthly values for temperature and precipitation, the whole of the Arabian Peninsula is classified as a hot desert climate (BWh). The climate is exceptionally hot, with continuous sunshine over the whole year.

A synthetic climate file for Dubai International Airport has been generated using Meteonorm 6.1 [24], containing hourly values for a whole year (Fig. 1). The average air temperature over the year is around $28{ }^{\circ} \mathrm{C}$. Maximum air temperature values are extremely high, reaching $45{ }^{\circ} \mathrm{C}$. Solar irradiation values are also very high, with maximum values close to $1000 \mathrm{~W} / \mathrm{m}^{2}$ over a horizontal surface. Summers are extremely hot and very humid, given the proximity to the sea.

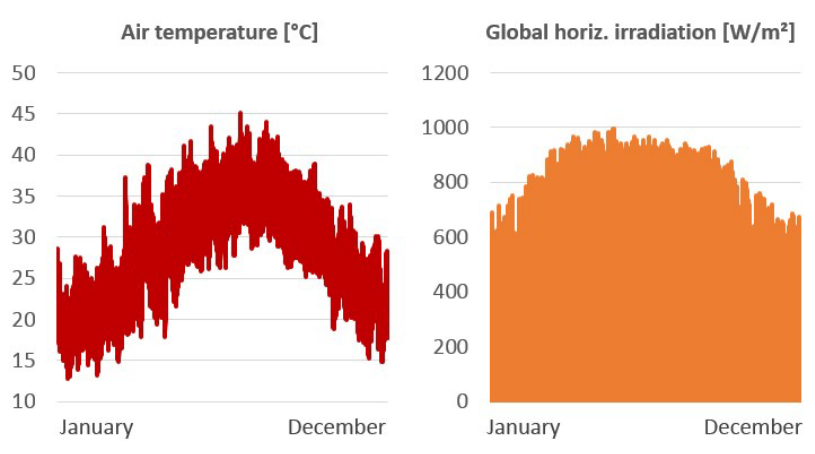

Fig. 1. Annual climate data for Dubai International Airport. 
An analysis of the evolution of temperatures over a typical day-night cycle has been performed by calculating 'average days' for every month. For this purpose, average temperatures at each hour of the day have been computed within each month. Results (Fig. 2) show a very regular pattern, with day-night temperature swings of around $10 \mathrm{~K}$ on average.

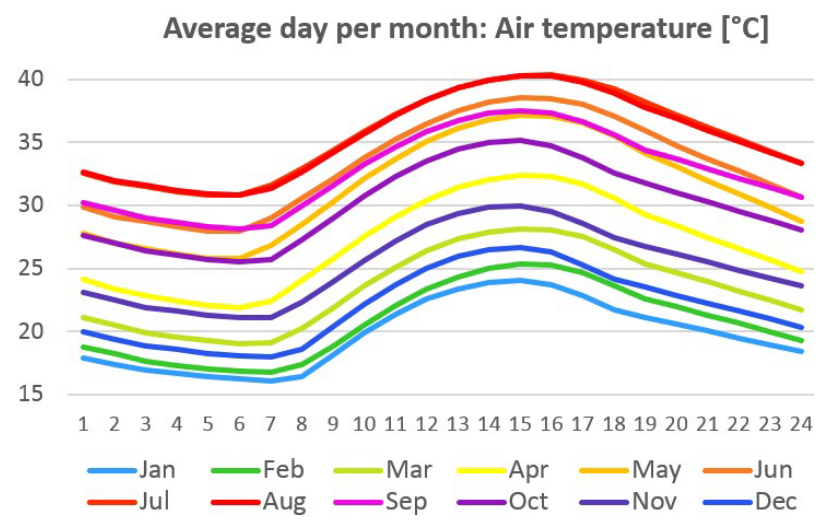

Fig. 2. Evolution of temperatures over an average day for each month, calculated from data for Dubai International Airport.

\section{Conceptual design}

The experimental facility comprises two sets of test chambers: one large 'hot hall' corresponding to outdoor weather conditions, and three 'cold chambers' corresponding to indoor comfort ambient conditions.

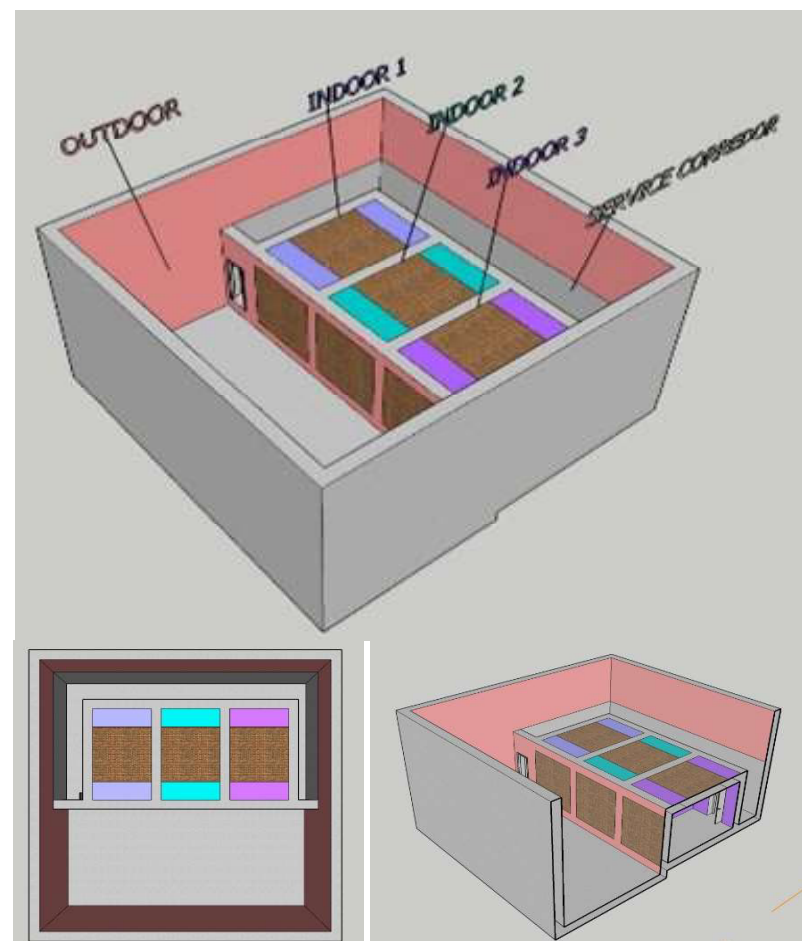

Fig. 3. Schematic and cross sections of the test facility.

Additionally, a service corridor is installed in the outer perimeter of cold chambers, to mitigate heat transfer towards the exterior and differences in heat loads among cold chambers.
The large hall is designed with an 'inverse L' cross section, so that two surfaces of the small chambers are exposed to the hall. This test setup allows to test wall or roof constructions under controlled transient conditions. Fig. 3 presents the schematics of the test facility.

The following main characteristics should be noted:

- Cold chambers are elevated above ground level to allow for the placement of ventilation diffusers below the test samples.

- The height of the hot hall is substantially larger than the height of the cold chambers, to keep stratification and non-homogeneous temperature distribution away from the test samples.

- The height of the hot hall allows sufficient free height on top of the cold chambers to allow for construction processes, elevation of goods, etc. as well as the avoidance of stratification effects.

- All envelope items are heavily insulated, particularly for cold chambers where calorimetric measurements will be performed.

\section{Experimental conditions}

The experimental set-up allows for the control of the boundary conditions at both sides of the construction elements to be tested. In this way, a variety of different situations can be tested. Three standard test scenarios are proposed: one stationary condition (5.1) and two dynamic conditions (5.2 and 5.3).

\subsection{Stationary condition}

Fixed temperatures are maintained at both sides of the tested element, and therefore the temperature difference across it remains constant. This test scenario is the fastest and most suitable for characterizing the steadystate thermal performance of a building envelope, which can be expressed as a thermal resistance ( $R$-value) or a thermal transmittance ( $U$-value).

For this test scenario, the standard boundary temperatures are $25{ }^{\circ} \mathrm{C}$ and $65{ }^{\circ} \mathrm{C}$, representing an indoor condition and an outdoor condition. There is thus a constant temperature difference of $40 \mathrm{~K}$ across the tested building component.

\subsection{Dynamic condition (sine wave)}

In this scenario, one of the boundary temperatures is fixed, while the other one fluctuates with a 24 hour period, intended to represent the day/night cycle. This scenario is a transient condition that captures the dynamic properties of the building envelope (time lag, thermal capacitance).

The standard boundary temperatures are $25{ }^{\circ} \mathrm{C}$ and 35-65 ${ }^{\circ} \mathrm{C}$, representing an indoor condition and a fluctuating outdoor condition. The outdoor temperature is defined as a sinusoidal curve with mean of $50{ }^{\circ} \mathrm{C}$, amplitude of $\pm 15 \mathrm{~K}$ and period of 24 hours. 


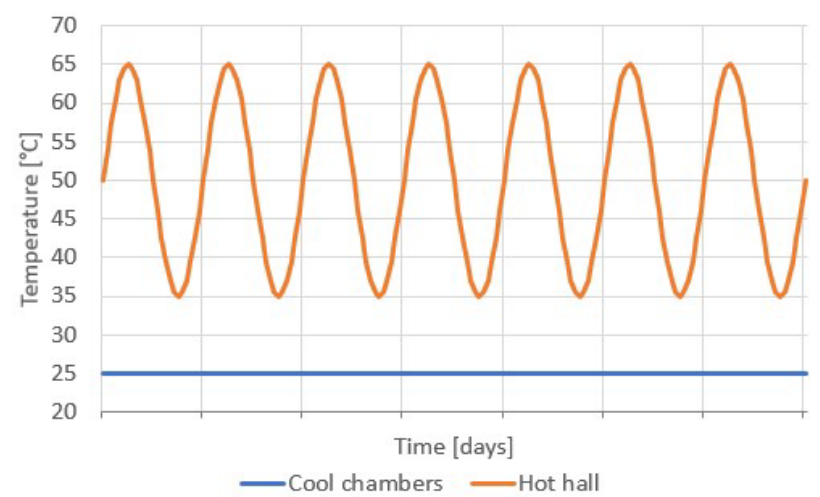

Fig. 4. Test scenario \#2: dynamic condition (sine wave).

\subsection{Dynamic condition (linear increment)}

In this scenario, one of the boundary temperatures is fixed, while the other one experiences a constant increment over time. This scenario is a transient condition but can be considered pseudo-stationary as it links two stationary states.

The standard boundary temperatures are $25^{\circ} \mathrm{C}$ and 35-65 ${ }^{\circ} \mathrm{C}$, representing an indoor condition and a fluctuating outdoor condition. In this case, the outdoor temperature initially keeps at $35^{\circ} \mathrm{C}$, then experiences a ramp increment of $2 \mathrm{~K}$ per hour, and keeps constant again once a temperature of $65^{\circ} \mathrm{C}$ is reached.

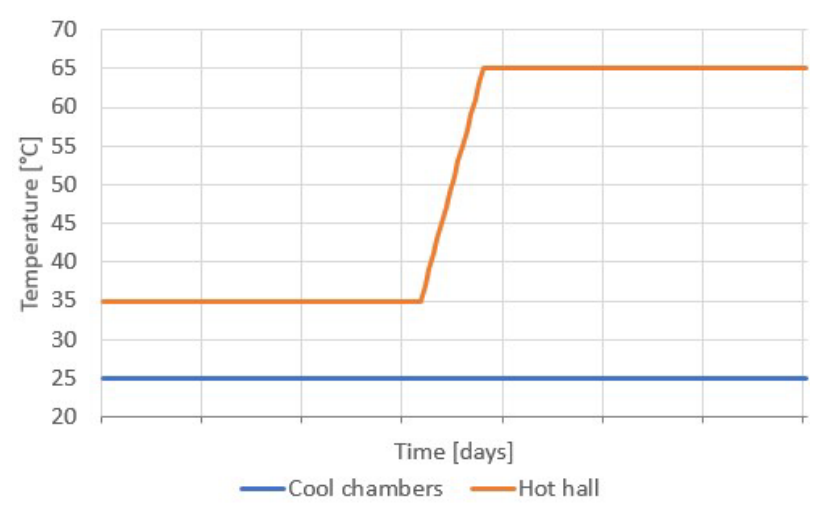

Fig. 5. Test scenario \#3: dynamic condition (linear increment).

\subsection{Stabilization and campaign length}

Based on previous numerical and experimental experience, a minimum experimental campaign duration of 7 days is prescribed.

- Transient numerical studies on building envelope heat transfer were performed in [25], simulating a series of walls with constant indoor temperature and fluctuating outdoor temperature with a period of $24 \mathrm{~h}$. Results from this study (Fig. 6) indicated that the time required for stabilization differed among scenarios, but a period of 7 days ensured a stable cycle (independently of previous conditions) in all cases assessed.

- With regards to experimental assessment procedures, no clear specification has been found in literature. In broad terms, the duration of the experimental campaign is defined by the required precision of the measurement.
In general, low temperature differences across the building component and higher insulation levels require longer experimental periods. The required duration for measurements is also influenced by the specific analysis method used, and often is not clearly stated in the standards (recommendations range from 72 hours to more than 7 days). Inductive campaign lengths can be deducted from [17]. This study concluded that dynamic methods achieved a reasonable precision within 7 days, but the average method in [8] only did so with a high temperature difference between boundary conditions.

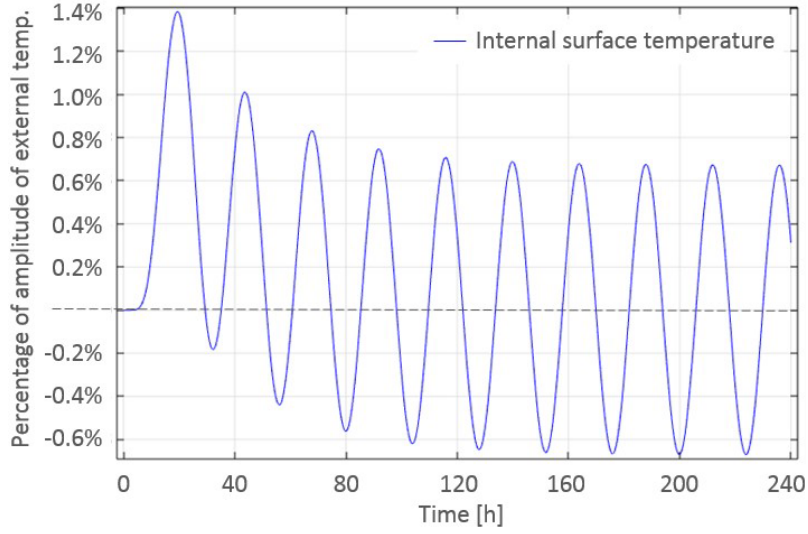

Fig. 6. Fluctuation of indoor surface temperature for a dynamic thermal simulation of an externally insulated wall with insulation thickness of $200 \mathrm{~mm}$.

\section{Monitoring layout}

The monitoring layout (Fig. 7) comprises the following: - Ambient temperature in each cold chamber (2 sensors), hot hall (10 sensors), and walkway (2 sensors).

- Ambient relative humidity in each cold chamber (1 sensor) and hot hall (2 sensors).

- Internal surface temperature of cold chambers: 4 units in each tested wall/roof, 1 unit in other walls and floor.

- External surface temperature of cold chambers: 4 units in each tested wall/roof.

- Heat flux through test walls/roofs of cold chambers: 3 sensors in each component.

- Thermal energy: 1 heat meter in each cold chamber.

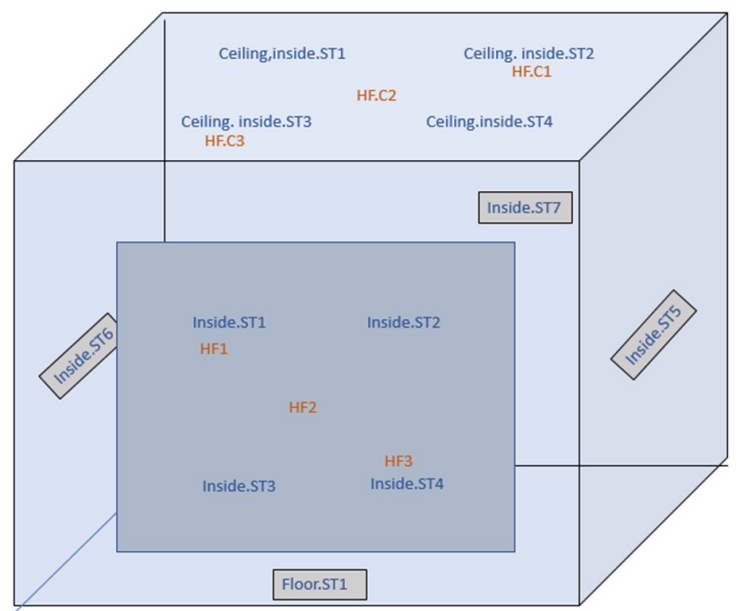

Fig. 7. Monitoring layout for a cold chamber. 


\section{Uncertainty assessment}

Uncertainty assessment is performed by monitoring heat flows through two individual measurements:

- Bounding surfaces of cold chambers

- Heat meters in cold chambers

A heat balance model is performed for each test chamber on a daily basis. As heat transfer through both measurements should be identical, the difference between both is assumed to be the uncertainty of the measurement. This uncertainty incorporates both energy flows through not properly measured items (e.g. convective heat transfer) and uncertainties associated to individual measurements (e.g. accuracy of heat meters).

The simplified zonal model is presented in Fig. 8.

\begin{tabular}{|c|c|c|c|c|}
\hline \multicolumn{5}{|c|}{$T_{0}$} \\
\hline $\mathrm{H}_{01}$ & & $\mathrm{H}_{02}$ & & $\mathrm{H}_{03}$ \\
\hline $\mathrm{T}_{1}$ & $\mathrm{H}_{12}$ & $\mathrm{~T}_{2}$ & $\mathrm{H}_{23}$ & $T_{3}$ \\
\hline $\mathrm{H}_{1 \mathrm{e}}$ & & $\mathrm{H}_{2 \mathrm{e}}$ & & $\mathrm{H}_{3 \mathrm{e}}$ \\
\hline & & $\mathrm{T}_{\mathrm{e}}$ & & \\
\hline
\end{tabular}

Key: 0 Walkway

1 Experimental chamber 1 (indoor conditions)

2 Experimental chamber 2 (indoor conditions)

3 Experimental chamber 3 (indoor conditions)

e Large chamber (outdoor conditions)

Fig. 8. Schematic drawing of heat transfer model.

The energy balance of test chambers is formulated in (1).

$$
\begin{aligned}
Q_{H V A C, i} & =H_{i e}\left(T_{e}-T_{1}\right)+H_{i 0}\left(T_{0}-T_{1}\right) \\
& +H_{i j}\left(T_{j}-T_{1}\right)+H_{i k}\left(T_{k}-T_{1}\right)+\varepsilon
\end{aligned}
$$

For each chamber (i), cooling load (HVAC) is formulated alongside heat transfer with the hot hall (e), the walkway $(0)$ and the other chambers $(j, k)$. For each of these paths, the heat transfer coefficient $(H)$ incorporates all subpaths (one-dimensional transfer through surfaces plus multi-dimensional heat transfer at junctions). Finally, an additional subpath $(\varepsilon)$ is added for non-measured, systematic deviations.

For test walls/roofs, heat transfer is monitored using heat flux meters, and thus $H_{\mathrm{ie}}$ is directly obtained. Combining this data with measured boundary temperatures, the $U$-value of such components can be obtained.

Heat transfer coefficients of the other envelope components, together with the systematic deviation term $\varepsilon$, are obtained by means of a steady-state calibration of the test rooms. The calibration is performed through a set of 8 experimental sequences, where a variety of temperature set points are imposed to each of the test chambers, so that proper identification of calibration coefficients can be achieved for all subpaths identified in (1) by means of linear regression methods.
This process is expected to be performed every time major changes are made to the infrastructure, such as the substitution of a wall and construction of a new one, modifying junctions or airtightness conditions. However, it is not required for minor changes such as the incorporation of insulation to test walls of chambers.

Considering the care taken in the design and construction of the experimental chambers, the large insulation levels in all components and the airtightness achieved, it is expected that calibration coefficients will be small.

\section{Present status \& future work}

The construction of the test facility finalized in 2019 . After commissioning tests of HVAC systems and sensors, the commissioning of the facility as an experimental system will be performed during Q1 2020. Calibration tests will be performed between February and March, followed by the initial experimental sequences in April. The facility is expected to be operative within Q2 2020, with R\&D tests already scheduled for that period.

\section{References}

1. UNEP, Sustainable Building and Climate Initiative, (2015)

2. E.U., 2002/91/EC, Energy Performance of Buildings (2002)

3. E.U., 2010/31/EU, Energy Performance of Buildings (recast) (2010)

4. ANSI/ASHRAE/IES Standard 90.1 (2019)

5. Government of Spain, Technical Building Code, CTE DB-HE (2019)

6. Government of Dubai, Municipality of Dubai, Al Safat, Dubai Green Building Evaluation System (2016)

7. ISO $6946: 2017$

8. ISO 9869-1:2014

9. ASTM C1155-95 (2013)

10. Abu Dhabi Urban Planning Council, Estidama, Pearl Building Rating System: Design \& Construction, Version 1.0 (2010)

11. General Headquarters of Civil Defence, UAE Fire and Life Safety Code of Practice (2011)

12. ISO 8302:1991

13. IEA EBC Annex 58, Inventory of full scale test facilities for evaluation of building energy performances (2016)

14. P. Wouters et al, Build. Environ. 4 (1993)

15. A.H. Deconinck et al, En. Build. 130 (2016)

16. R. Garay et al, En. Build. 147 (2017)

17. R. Garay, Universitat de Lleida https:/www.tdx.cat/handle/10803/460756

18. I.A. Atsonios et al., En. Build. 154 (2017) 
19. IEA EBC Annex 58

20. IEA EBC Annex 71

21. Research Infrastructures on Energy Efficiency in Buildings, EU Grant agreement ID 262476

22. www.sifs.me

23. ISO $13786: 2017$

24. www.meteonorm.com

25. Breakthrough solutions for adaptable envelopes for building refurbishment, EU Grant agreement ID 637186 